\title{
Medical Image Compression Using Wavelets
}

\author{
K Gopi ${ }^{1}$, Dr. T. Rama Shri ${ }^{2}$ \\ ${ }^{I}$ Asst. Prof., Department of ECE, SITAMS, Chittoor, India \\ 2. Prof., Department of ECE, S.V.University College of Engineering, SVU, Tirupati, India
}

\begin{abstract}
With the development of CT, MRI, PET, EBCT, SMRI etc, the scanning rate and distinguishing rate of imaging equipment is enhanced greatly. Using wavelet technology, medical image can be processed in deep degree by denoising, enhancement, edge extraction etc, which can make good use of the image information and improve diagnosing. Compressions based on wavelet transform are the state-of-the-art compression technique used in medical image compression. For medical images it is critical to produce high compression performance while minimizing the amount of image data so the data can be stored economically. Modern radiology techniques provide crucial medical information for radiologists to diagnose diseases and determine appropriate treatments. Such information must be acquired through medical imaging (MI) processes. Since more and more medical images are in digital format, more economical and effective data compression technologies are required to minimize mass volume of digital image data produced in the hospitals. The wavelet-based compression scheme contains transformation, quantization, and lossless entropy coding. For the transformation stage, discrete wavelet transform and lifting schemes are introduced. In this paper an attempt has been made to analyse different wavelet techniques for image compression. Hand designed wavelets considered in this work are Haar wavelet, Daubechie wavelet, Biorthognal wavelet, Demeyer wavelet, Coiflet wavelet and Symlet wavelet. These wavelet transforms are used to compress the test images competitively by using Set Partitioning In Hierarchical Trees (SPIHT) algorithm. SPIHT is a new advanced algorithm based on wavelet transform which is gaining attention due to many potential commercial applications in the area of image compression. The SPIHT coder is also a highly refined version of the EZW algorithm.
\end{abstract}

Keywords - Wavelet, Medical Image, Compression, PSNR

\section{INTRODUCTION}

Image compression is one of the most important and successful applications of the wavelet transform. Mature wavelet based image coders like the JPEG2000 standard [1] are available, gaining popularity, and easily outperform traditional coders based on the discrete cosine transform (DCT) like JPEG [2]. Unlike in DCT based image compression, however, the performance of a wavelet based image coder depends to a large degree on the choice of the wavelet. This problem is usually handled by using standard wavelets that are not specially adapted to a given image, but that are known to perform well on photographic images.

However, many common classes of images do not have the same statistical properties as photographic images, such as fingerprints, medical images, scanned documents and satellite images. The standard wavelets used in image coders often do not match such images resulting in decreased compression or image quality. Moreover non-photographic images are often stored in large databases of similar images, making it worthwhile to find a specially adapted wavelet for them.

Memory and bandwidth are the prime constraints in image storage and transmission applications. One of the major challenges in enabling mobile multimedia data services will be the need to process and wirelessly transmit a very large volume of data. While significant improvements in achievable bandwidth are expected with future wireless access technologies, improvements in battery technology will lag the rapidly growing energy requirements of future wireless data services.

One approach to mitigate this problem is to reduce the volume of multimedia data transmitted over the wireless channel via data compression techniques. This has motivated active research on multimedia data compression techniques such as JPEG [3, 4], JPEG 2000 [5, 6] and MPEG [7]. These approaches concentrate on achieving higher compression ratio without sacrificing the quality of the image. However these efforts ignore the energy consumption during compression and RF transmission. Since images will constitute a large part of future wireless data, the thesis aim on developing energy efficient and adaptive image compression and communication techniques. Based on wavelet image compression, energy efficient multi-wavelet image transform is a technique developed to eliminate computation of certain high-pass coefficients of an image. 


\section{RELATED WORK}

Any type of data transmission or storage without compression is impractical for the following reasons.

- The data handled by different digital environments is increasing at a rate more than twice a year.

- Similar to the case of storage, the transmission is the other concern. With the insight into the World Wide Web (WWW), where more than 7500 Tera Bytes of data is being downloading and/or uploading, the transmission is the major concern in the modern world.

The storing of digital data and transmission without compression would be a tragedy. Hence it was made mandatory to have compression in one form or other. To incorporate compression or even to modify any image in any sense, frequency domain is assumed to be more convenient. To transform an image from spatial domain to frequency domain, the basic technique that was in use is Discrete Cosine Transform (DCT). DCT is very simple technique with low complexity. But it has the following pitfall.

- Only spatial correlation of the pixels inside the single 2D block is considered, and the correlation from the pixels of the neighboring blocks is neglected. It results blocking artifacts. If it so, the human interpreter may not identify image properly.

After the mention of Wavelet by Haar in his Ph.D thesis in 1909, Discrete Wavelet Transform (DWT) was used in most of the image compression applications as it overcomes the disadvantages of DCT. But it too has the following limitations.

- Computational Complexity

- Memory Complexity

- Low image quality

- Floor operating losses

- Poor compression ratio

- Low bit degradation

Wavelet transforms have received significant attention in many fields, such as mathematics, digital signal and image processing, because of their ability to represent and analyze data. The wavelet transform, defined by Yves Meyer and J. Lemarie offers good localization in both, space and frequency domains and can be implemented by fast algorithms. Since the discrete wavelet transform (DWT) was presented by Mallat, many researchers on signal analysis and image compression have derived fruitful results due to its well time-frequency decomposition. Recently, a new wavelet construction called lifting scheme, has been developed by Wim Sweldens and Ingrid Daubechies [8]. It has other applications, such as the possibility of defining a wavelet-like transform that maps integers to integers [9]. This method has gained increasing interest in scientific community, due to its reduced computational complexity by first factoring a classical wavelet filter into lifting steps.

\section{DESIGN METRICS}

Digital image compression techniques are examined with various metrics. Among those the most important one is Peak Signal to Noise Ratio (PSNR) which will express the quality. There exists another property which expresses the quality, that is, Mean Square Error (MSE). PSNR is inversely proportional to MSE. The other important metric is Compression Ratio, which express the amount of compression embedded in the technique. In theory, it was observed that PSNR and Compression ratios are inversely related. The other metrics are Encoding Time, Decoding Time and Transforming Time.

\subsection{Mean Square Error}

Among the quantitative measures, a class of criteria used often is called the mean square criteria. It refers to some sort of average or sum (or integral) of squares of the error between two images. MSE for monochrome image

$$
\frac{1}{N^{2}} \sum_{i}^{N} \sum_{j}^{N}(X(i, j)-Y(i, j))^{2}
$$

MSE for color image

$$
\frac{1}{N^{2}} \sum_{i}^{N} \sum_{j}^{N}\left[\left(r(i, j)-r^{*}(i, j)\right)^{2}+\left(g(i, j)-g^{*}(i, j)\right)^{2}+\left(b(i, j)-b^{*}(i, j)\right)^{2}\right]
$$

where $r(i, j), g(i, j)$ and $b(i, j)$ represents a color pixel in location $(i, j)$ of the original image, $r^{*}(i, j), g^{*}(i, j)$ and $b^{*}(i, j)$ represents color pixel of the reconstructed image and N X N denotes the size of the pixels of these color images. where $\sigma^{2}$ is the variance of the desired image and $\sigma_{e}^{2}$ is average variance.

\subsection{Peak Signal to Noise Ratio}


Peak Signal to Noise Ratio is defined as the ratio between signal variance and reconstruction error variance. Mean Square Error, Peak Signal to Noise Ratio and Compression Ratios are calculated from the following expressions.

\subsection{Compression Ratio}

$$
\mathrm{PSNR}=10 \log _{10} \frac{255^{2}}{M S E}
$$

Compression ratio is defined as the ratio between the original image size and compressed image size.

$$
\text { Compression Ratio }=\frac{\text { Original }- \text { image }- \text { size }}{\text { Compressed }- \text { image }- \text { size }}
$$

\subsection{Encoding Time, Decoding Time and Transforming Time}

Any compression system uses one of the encoding techniques to encode the input information. The encoding operation is very crucial for the success of the compression system. It involves the representation of the input information in a form suitable for storage and transmission. The time required to perform this operation is referred to as encoding time. The reverse process to encoding is decoding and the corresponding time required to decode an encoded data is decoding time. In general, the information to be compressed will be represented in time or spatial domain. To compress the data, it was observed that it is convenient to represent the data in frequency domain. Hence the information in time domain needs to be converted into frequency domain. For that, one of the transforming techniques will be used. Again it involves some consumption of time. This time is referred to as transforming time. These times are measured in seconds.

\subsection{Haar wavelet}

\section{TRADITIONAL WAVELETS}

Haar Wavelet is defined by

$$
\psi(x)=\left\{\begin{array}{c}
1, x \varepsilon[0,1 / 2) \\
-1, x \varepsilon[1 / 2,1) \\
0, \text { otherwise }
\end{array}\right.
$$

In 1910, Haar already used this function for constructing an orthonormal basis in L2(IR) by means of dilations and integer translations of a so-called mother function. Besides the orthonormality of a basis, Haar's concept coincides with the wavelet transform and its inversion formula. The Haar wavelet transform can be interpreted as a multiscale differential operator of order 1. The Haar wavelet is also the simplest possible wavelet. The technical disadvantage of the Haar wavelet is that it is not continuous, and therefore not differentiable. This property can, however, be an advantage for the analysis of signals with sudden transitions, such as monitoring of tool failure in machines.

\subsection{Daubechie wavelet}

The wavelet transform is also easy to put into practice using the fast wavelet transform. Daubechies wavelets are widely used in solving a broad range of problems, e.g. self-similarity properties of a signal or fractal problems, signal discontinuities, etc. In 1988 Ingrid Daubechies [10] constructed the entire class of orthonormal wavelet bases $\mathrm{Dbr}, r==1,2, \ldots$, of compactly supported functions using the analogy with the maxflat filters of signal theory. The choice of the order $r$ defines various Daubechies wavelets that represent a new family of special functions. In general the Daubechies wavelets are chosen to have the highest number A of vanishing moments, (this does not imply the best smoothness) for given support width $\mathrm{N}=2 \mathrm{~A}$, and among the $2^{\mathrm{A}-1}$ possible solutions the one is chosen whose scaling filter has extremal phase. Except the Haar wavelet Dbl (formula 3.35) Daubechies wavelets have no explicit expressions and can only be calculated through recursion. Daubechies wavelet is more efficient than Haar in analysis and colligation.

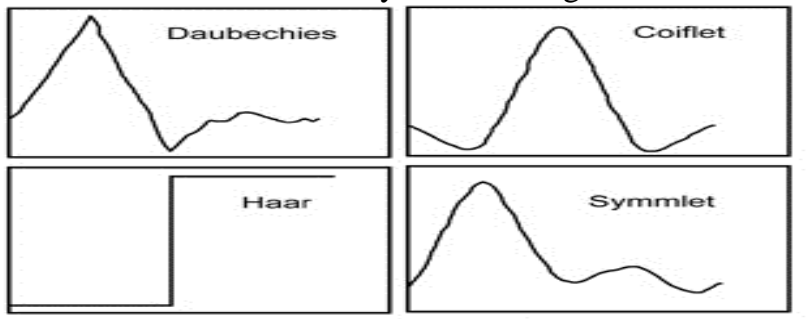

Figure 1. Basis Functions of Different Wavelets. 


\subsection{Biorthogonal Wavelet}

The orthogonality of a wavelet basis is a very restrictive request, it cannot be fulfilled for the odd number of dilatation equation coefficients, e.g. the linear spline and matched wavelet. Besides orthogonal wavelet bases have very strong asymmetry that is an undesirable property in many applications. If we give up of the requirement that the same orthogonal basis is used for the decomposition (analysis) and the reconstruction (synthesis), symmetry is possible. The biorthogonal bases can commute roles, so that the tilde functions are used in analysis while the dual ones are used in synthesis. The choice depends on the regularity and number of vanishing moments for both. It is a well-known fact in the filter theory community that symmetry and perfect reconstruction are incompatible (except for the Haar wavelet) when the same FIR filters are used for decomposition and for reconstruction process. To circumvent this difficulty two wavelets are introduced instead of one.

\subsection{Coiflet wavelet}

They were constructed by Ingrid Daubechies as requested by her colleague Ronald Coifman, whom they were named after. In order to calculate the initial coefficient sequence of the pyramidal algorithm as simply as possible, it is useful to have moments of the scaling function of as high an order as possible equal to zero. They are less asymmetrical than the wavelets Dbr. Relative to the support length, Coifr is comparable to the wavelets $\mathrm{Db} 3 \mathrm{r}$ and Sym3r, while relative to the number of vanishing moments it is comparable to the wavelets $\mathrm{Db} 2 \mathrm{r}$ and Sym2r. They are used in numerical analysis. The wavelet is near symmetric, their wavelet functions have $N / 3$ vanishing moments and scaling functions $N / 3-1$, and has been used in many applications using Caldron-Zygmund Operators. Both the scaling function (low-pass filter) and the wavelet function (High-Pass Filter) must be normalised by a factor $\frac{1}{\sqrt{2}}$. Below are the coefficients for the scaling functions for C6-30. The wavelet coefficients are derived by reversing the order of the scaling function coefficients and then reversing the sign of every second one (ie. C6 wavelet $=\{-0.022140543057,0.102859456942,0.544281086116$, $-1.205718913884,0.477859456942,0.102859456942\})$. Mathematically, this looks like $B_{k}=(-1)^{k} C_{N-1-k}$ where $k$ is the coefficient index, $B$ is a wavelet coefficient and $C$ a scaling function coefficient. $N$ is the wavelet index, ie 6 for C6.

\subsection{Symlet wavelet}

Symlet wavelets (Symr) represent a modification of the Daubechies wavelets, done to improve their symmetry. Still, to retain the simplicity of the Daubechies wavelets, they are only almost symmetrical. They are also constructed by the coefficients of the frequency response as there are various ways to group the function to the factors $\hat{h}(w)$ and $\overline{\hat{h}(w)}$. By the choice of the frequency response $\hat{h}(w)$ so that all of its roots by module are smaller than or equal to one, we arrive at the Daubechies wavelet Dbr. By a different choice we arrive to the more symmetry wavelet Symr.

Thus the other properties of these wavelets are similar to the Dbr wavelet properties. A full symmetry cannot be achieved within the frame of the orthonormal wavelet basis with a finite support, other than the Haar Dbl wavelet.

\subsection{Demeyer Wavelet}

By defining the wavelet and scaling function in the frequency domain by functions with a compact support but a greater smoothness, the scaling function and wavelet can belong to the space Coo and can decrease faster than the polynomial. Both the wavelet and the scaling function are constructed in the frequency domain using trigonometric functions, but so that their Fourier transforms have compact support. The scaling function is symmetrical around point 0 , while the wavelets are symmetrical around point $1 / 2$. The scaling function and wavelet do not have compact supports, but they decrease faster than any" inverse polynomial". This wavelet is an infinitely differentiable function.

\section{Simulation RESUlTS}

In this section the simulation results of the said techniques are presented, which are obtained after implementing those in MATLB. The design metrics considered are already presented in the previous sections. The compression ratio should be maintained with considerable quality or in other way; PSNR should be maintained with sufficient size reduction in the memory point of view. The GUI used in the work was given in the figure 2. Wide range of medical images, including both MRI and CT scan images were considered. The algorithms are implemented in MATLAB. The GUI used in the work was given in the figure 1. The input 
images are shown in figure 2. The table 1 gives the performance of hand designed wavelet transform on the input images. The performance of these wavelet transforms was analyzed and plotted in figure 3.

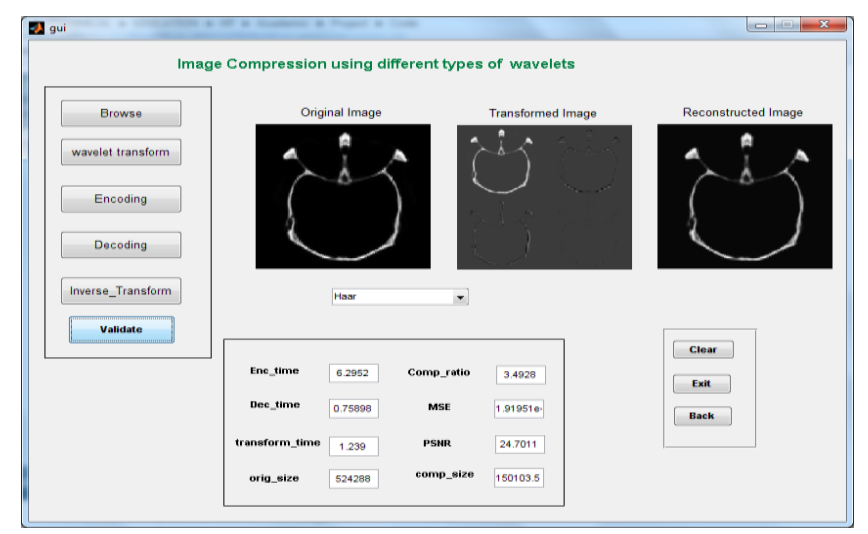

Fig. 2. GUI used in MATLAB

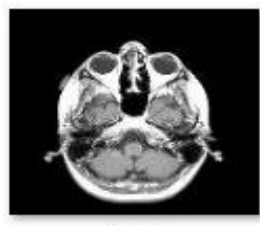

MRI-1

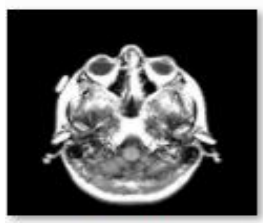

MRI-4

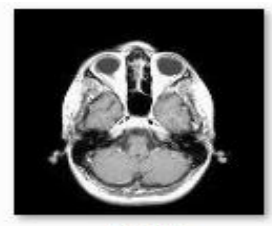

MRI-2

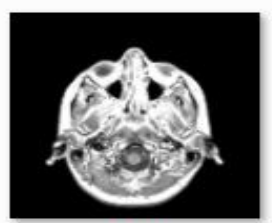

MRI-5

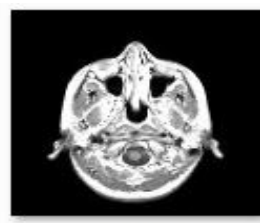

MRI-3

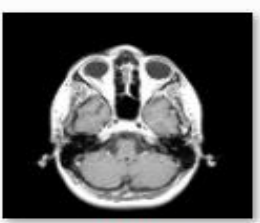

MRI-6

Fig. 3. Input MRI images

Table 1. Performance of different wavelet transforms on medical images

\begin{tabular}{|c|c|c|c|c|c|c|c|}
\hline \multirow{2}{*}{ Image } & Parameter & Haar & Daubechie & $\begin{array}{c}\text { Biorthogona } \\
\text { 1 }\end{array}$ & Demeyer & Coiflet & Symlet \\
\hline \multirow{2}{*}{ CT } & CR (bpp) & 3.4 & 1.77 & 3.05 & 1.9 & 2.89 & 1.88 \\
\cline { 2 - 8 } & PSNR (dB) & 24.7 & 24.3 & 26.7 & 24.6 & 45.5 & 24.6 \\
\hline \multirow{2}{*}{ MRI-1 } & CR (bpp) & 3 & 2.6 & 2.7 & 1.62 & 2.4 & 2.7 \\
\cline { 2 - 8 } & PSNR (dB) & 24.8 & 30.9 & 32.18 & 30.8 & 49.8 & 30.9 \\
\hline \multirow{2}{*}{ MRI-2 } & CR (bpp) & 2.9 & 2.6 & 2.7 & 1.61 & 2.4 & 2.7 \\
\cline { 2 - 8 } & PSNR (dB) & 25.2 & 31 & 32.8 & 31.3 & 50 & 31.5 \\
\hline \multirow{2}{*}{ MRI-3 } & CR (bpp) & 3.62 & 3.478 & 3.53 & 2.12 & 3.2 & 3.57 \\
\cline { 2 - 8 } & PSNR (dB) & 23.1 & 23.74 & 24.07 & 23.77 & 40.0 & 23.7 \\
\hline \multirow{2}{*}{ MRI-4 } & CR (bpp) & 3.03 & 2.6 & 2.73 & 1.63 & 2.4 & 2.72 \\
\cline { 2 - 8 } & PSNR (dB) & 24.2 & 30.54 & 31.39 & 30.4 & 49.2 & 30.4 \\
\hline \multirow{2}{*}{ MRI-5 } & CR (bpp) & 3.05 & 2.6 & 2.7 & 1.63 & 2.46 & 2.73 \\
\cline { 2 - 8 } & PSNR (dB) & 23.9 & 30.1 & 31 & 30.05 & 48.8 & 30.42 \\
\hline \multirow{2}{*}{ MRI-6 } & CR (bpp) & 3.05 & 2.69 & 2.75 & 1.64 & 2.47 & 2.75 \\
\cline { 2 - 8 } & PSNR (dB) & 24.7 & 30.87 & 32.44 & 30.62 & 50.1 & 30.91 \\
\hline
\end{tabular}




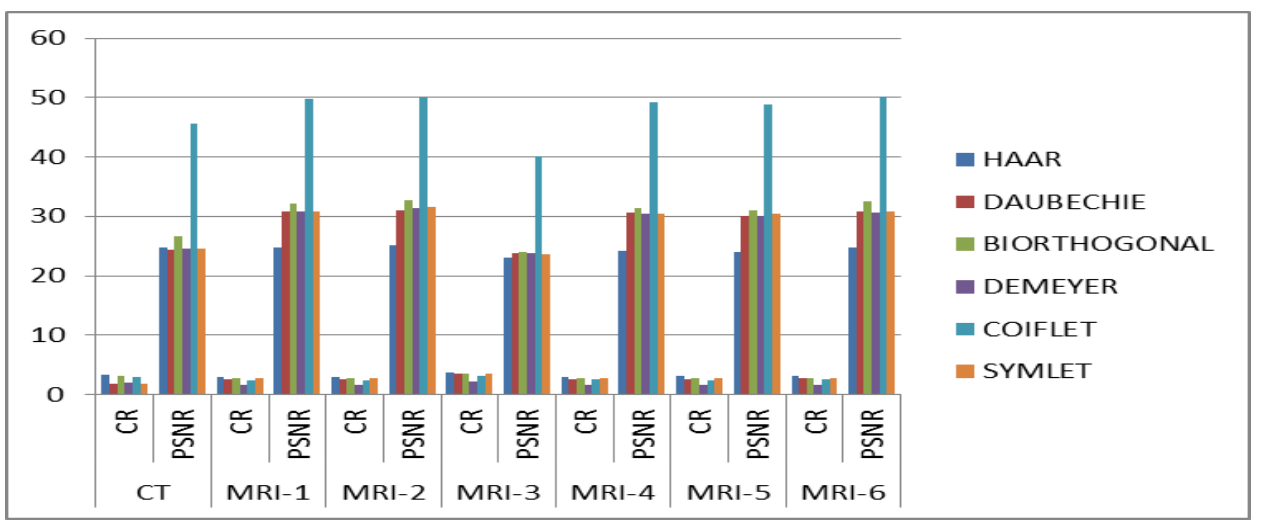

Fig. 4. Performance of different wavelet transforms on medical images

\section{Conclusions}

In this paper, the performance of Hand designed wavelets is presented. Haar wavelet, Daubechie wavelet, Demeyer wavelet, Coiflet wavelet and Symlet wavelets are considered under Hand designed wavelet category. Except Coiflet wavelet, all the Hand designed wavelets produced less PSNR around 30dB and less compression ratio around $2 \mathrm{bpp}$. Coiflet wavelet produced high PSNR around $47 \mathrm{~dB}$, but at low compression ratio in the ranges of only $2 \mathrm{bpp}$.

\section{Journal Papers:}

\section{REFERENCES}

[1] I. JTC1/SC29/WG1, "JPEG 2000 - lossless and lossy compression of continuous- tone and bi-level still images", Part 1: Minimum decoder. Final committee draft, Version 1.0., March 2000.

[2] G.Wallace, "The JPEG still picture compression standard”, IEEE TCE, 38, 1992.

[3] Independent JPEG Group, version 6a:http://www.ijg.org.

[4] G. K. Wallace, "The JPEG still picture compression standard", in IEEE Transactions on Circuits and Systems for Video Technology, vol. 6, June 1996.

[5] O. K. Al-Shaykh, "JPEG-2000: A new still image compression standard", in Conference Record of Thirty-Second Asilomar Conference on Signals Systems and Computers, vol. 1, pp. 99-103, 1998.

[6] JPEG2000, http://www.jpeg.org/JPEG2000.htm.

[7] Moving Picture Expert Group Standard, http://www.mpeg. org/MPEG/index.html.

[8] W. Sweldens, 1997, "The lifting scheme: A construction of second generation wavelets", SIAM J. Math. Anal., Vol.29 (2), pp.511546.

[9] I. Daubechies, W. Sweldens, 1998, "Factoring wavelet into lifting steps", J. Fourier Anal. Appl., Vol.4 (3), pp.247-269.

[10] Daubechies I., Orthonormal bases of compactls] supported wavelets, Comm.Pure Appl. Math. 41, 909-996 (1988)

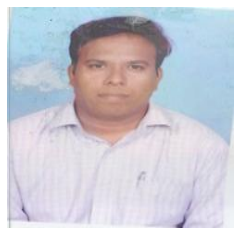

K.Gopi is working as an Assistant Professor in Department of ECE, Sreenivasa Institute of Technology and Management Studies, chittoor. He has 9 years of teaching experience. He has done his M.E in applied electronics in the year 2007. He is currently doing his research in digital image processing in the field of Medical Image Compression in S.V. University, Tirupati.

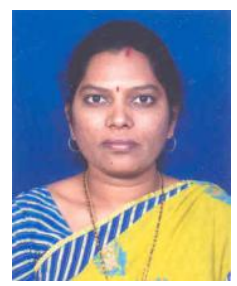

Dr.T.Rama Shri is working as Professor in the Department of ECE, S.V University College of Engineering, Tirupati. She has more than 18 years of Teaching Experience. She has published more than ten research papers in national and international conferences. Her research interests include Digital Image Processing. She is a life member of ISTE and IETE. 